\title{
UM ANALISADOR DE ESPECTRO COM ARDUINO NA FAIXA DE RÁDIO FM PARA FINS DIDÁTICOS
}

DOI: 10.37702/2175-957X.COBENGE.2021.3503

Vitor Bremgartner da Frota - vitorbref@ifam.edu.br Instituto Federal do Amazonas

Av Umberto Calderaro 670

69057-015 - Manaus - AM

harryson barbosa palheta - harryson.palheta@gmail.com

Instituto Transire

Rua Santos Dumont 125

69093-273 - Manaus - AM

Resumo: Este artigo apresenta o desenvolvimento de um software, chamado Plot Spectrum, que simula um Analisador de Espectro para fins didáticos em disciplinas de curso de Telecomunicações feito com Arduino e uma shield TEA5767 de rádio FM na frequência de 87,5 a $108 \mathrm{MHz}$ interagindo com um computador pessoal qualquer. Além disso, são apresentadas as funcionalidades do projeto comparando-as com um Analisador de Espectro real, ou um Osciloscópio com função compatível, e compara também com projetos semelhantes já publicados.

Palavras-chave: Analisador de Espectro. Arduino. Simulador. 


\section{UM ANALISADOR DE ESPECTRO COM ARDUINO NA FAIXA DE RÁDIO FM PARA FINS DIDÁTICOS}

\section{INTRODUÇÃO}

Muitas vezes, em disciplinas do curso de Telecomunicações, como Micro-ondas, Ondas e Antenas, entre outras que fazem estudos de sinais, utilizamos o analisador de espectro, instrumento que serve para a análise de sinais alternados no domínio da frequência. Nele é possível efetuarmos medidas de modulação, distorção e ruído. Operar esses equipamentos requer muita atenção, leitura dos manuais de operação, treinamentos e entendimentos de conceitos físicos como frequência, potência, tensão e período para que não haja equívocos no seu manuseio. Ter a leitura desses dados em uma tela digital colorida torna a experiência de aprendizagem muito mais atrativa, encurtando a dificuldade existente em disciplinas que utilizem esses equipamentos. Em uma breve pesquisa nas literaturas a respeito da utilização do Analisador de Espectro e em lojas especializadas, percebemos que o preço desse equipamento com suas funcionalidades básicas custa em torno de $R \$ 1.300,00$, como podemos ver no modelo Hantek DSO8060 5-em-1 Handheld no site http://www.banggood.com. Enquanto um com funcionalidades completas e de marca conhecida no segmento de eletrônica custa em torno de USD 24.760,00, como é o caso do Tektronix MDO4104C (TEKTRONIX, 2021).

A obtenção dos equipamentos em uma instituição de ensino não é algo simples, pois dependem de vários processos burocráticos que levam tempo a serem concluídos, se aprovados, além de recursos financeiros. Em alguns casos até ultrapassa o período (6 meses) de uma disciplina. O que nos traz a alternativa de fazer a própria ferramenta, são os chamados "makers", elaborando-a de forma mais rápida, podendo-se pedir os componentes através da Internet e estes itens chegando em torno de duas semanas a um mês, ou até dois meses, pedindo em alguns sites chineses. Os Makers provêm 'Movimento Maker' que tem a filosofia do "Do-It-Yourself", que em inglês quer dizer "Faça você mesmo". O que geralmente é feito por um custo menor que as ferramentas originais e às vezes com alguns recursos limitados, embora funcionais. Para essa tarefa de "faça você mesmo", existem várias tecnologias disponíveis no mercado para elaboração de circuitos eletrônicos como resistores capacitores, transistores, diodos, LEDs, placas de cobre, fontes de alimentação, etc... Existem também circuitos prontos como Arduino, Raspiberry $\mathrm{Pi}$, Orange $\mathrm{Pi}$ e muitos outros que ajudam nas práticas de automação.

Além dessa disponibilidade no mundo da eletrônica, a confecção dos próprios instrumentos recebe amplo apoio das comunidades na Internet.

Para confecção do próprio analisador de espectro neste trabalho, devemos seguir etapas que nos ajudam a entender como o mesmo funciona. Etapas como plotagem de gráfico, coleta dos dados, criação da interface, interação com a interface, setagem de valores e configurações, permitindo melhor simular um equipamento real, ou chegar 0 mais perto disso possível. Tais etapas são descritas nesse trabalho. Logo após os métodos de fabricação do software e comunicação dos dispositivos, foram feitos experimentos com um analisador real em comparação com o projeto criado para validar seu funcionamento, uso e competência. Por fim, após os conceitos, a metodologia, trabalhos relacionados e experimentos realizados, é possível descobrir se um analisador de espectro feito com as próprias mãos está apto para fins didáticos. 


\section{REFERENCIAL TEÓRICO}

\subsection{Analisador de Espectro}

O analisador de espectro é um instrumento de medição através do qual os sinais elétricos podem ser avaliados em determinadas frequências (FERRER, 2019). Através de uma tela, os analisadores mostram os componentes espectrais encontrados na entrada de um sinal elétrico, óptico ou acústico. Com o equipamento configurado corretamente, o sinal e a potência podem ser exibidos. No eixo vertical, vemos o conteúdo espectral do sinal em dB, e na horizontal, a frequência. Essa análise é baseada na revelação de todos os componentes. Ele também tem a capacidade de modelar luz, ondas de rádio e som, que podem ser combinados ou sobrepostos. O modelo mostrado na Figura 1, MDO 3014, da Tektronix, usado para este projeto, é um osciloscópio com a função de analisador de espectro, mas que atende plenamente as funções básicas requeridas, e tem a precisão muito próxima de um analisador de espectro como propriamente dito. Se tratando de interpretar o display de um analisador, temos destaques de alguns elementos como transformada rápida de Fourier (FFT), frequência total analisada (SPAN), faixa, frequência central, resolution bandwidth (RBW), escala, nível de referência e marcadores.

Figura 1 - Osciloscópio Tektronix MDO3014.

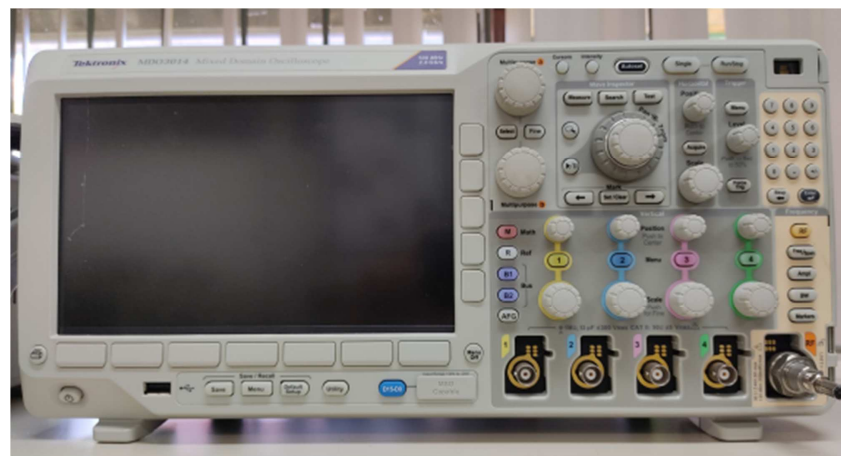

Fonte: Autores.

\subsection{Trabalhos Relacionados}

A seguir são citados três trabalhos relacionados onde serão comparados, com as semelhanças e diferenças com este aqui desenvolvido, mais a frente nos Resultados, após os Experimentos.

O primeiro, nota-se semelhante, não específico para a análise da frequência, mas também voltado para a aprendizagem, é um dos artigos publicados em 2016 pelo COBENGE, consiste em aprimorar o ensino da teoria de Processamento Digital com uma interface amigável usando MATLAB no domínio do tempo e da frequência (GUEDES; GÓES; SILVA, 2016). Nesse trabalho de 2016, foi desenvolvida três interfaces para consolidar, na prática, aulas teóricas da disciplina de Processamento de Sinais, onde o usuário pode acompanhar graficamente e escolher o tipo da análise (tempo ou frequência) do sinal em questão. É utilizado o GUIDE2 do MATLAB para desenvolver as interfaces de interação com o usuário, configurando parâmetros para a compreensão de assuntos como amostragem, transformada de Fourier, filtros e janelamento.

O segundo trabalho do COBENGE, de 2018, parecido com o anterior, facilitando também a compreensão de disciplinas difíceis para curso de engenharia, é sobre o desenvolvimento de ferramenta que possibilita o estudo de modulações utilizando também o MATLAB (FRANCHI et al., 2018). A didática deste consiste em aplicar 
conceitos como sinal modulante, portadora e sinal modulado de forma visual. É possível configurar os parâmetros e as fórmulas do sinal desejado. Cada sinal fica em gráficos separados na interface. Além disso, há um relatório que explica com mais detalhes que ocorrem na tela.

E por ultimo, temos o terceiro, e mais atual, trabalho, de 2019, é também do COBENGE, sobre um projeto multidisciplinar de um analisador de espectro com banco de filtros e subamostragem (MELO et al., 2019), que utiliza-se de recursos de um analisador para difundir conhecimento em várias matérias que utilizem este equipamento no curso de engenharia eletrônica. É utilizado também conversores A/D (analógico para digital) de baixo custo em paralelo. Além disso, possui filtros, simulações e altas taxas de amostragem com melhorias na captura. Para prover todos esses recursos, as simulações são feitas no MATLAB. Os procedimentos de decomposição e recomposição do sinal, onde é apresentado 7 blocos (etapas) de filtros. Na amostra apresentada são coletados 20 canais analógicos de faixas diferentes onde comprimem para um único canal e recompõe os 20 canais após passarem pelo sistema somador transformando-os em onda digital (quadrada) conversores A/D (analógico para digital) de baixo custo em paralelo.

\section{Materiais e Métodos Utilizados}

O trabalho de pesquisa compreende um estudo de caso, do software simulador desenvolvido, nomeado de Plot Spectrum, analisado no laboratório de medições do Instituto Federal do Amazonas (IFAM) - Campus Manaus Distrito Industrial (CMDI), curso de Tecnologia em Sistemas de Telecomunicações, onde utiliza-se o instrumento Osciloscópio Tektronix MDO3014 (100MHz/ 2.5G/s) com função de Analisador de Espectro, o qual foi usado como equipamento modelo para comparação. O simulador foi elaborado utilizando uma interface bastante similar a de um analisador de espectro, implementando-se o software com tecnologias WEB integrando-o com o hardware em Arduino e a shield TEA5767 até a satisfação das funcionalidades básicas.

A lista de componentes usados neste projeto consiste em:

- 1 Placa Arduino Uno

- 1 Shield TEA5767

- 1 cabo usb/speed

- 1 computador pessoal (Notebook Ideapad 320)

- 1 Osciloscópio com função de analidador (Tektronix MDO 3014)

- 1 Gerador de Sinais (Tektronix AFG 3101)

- 2 antenas com entrada de 50 ohms com conector $N$ fêmea

Podemos ver o esquema da montagem do circuito do simulador para o seu funcionamento na frequência suportada de 87,5 a $108 \mathrm{MHz}$ na Figura 2.

Figura 2 - Montagem do Arduino com a Shield TEA5767. 


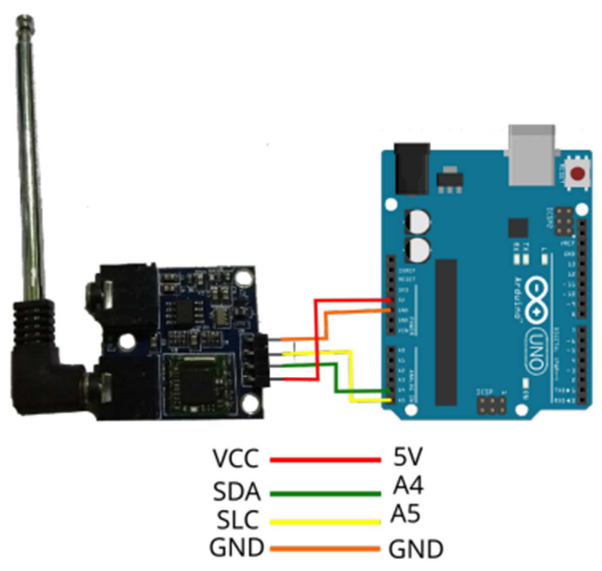

Fonte: Autores.

O esquema da Figura 2 foi conectado a um notebook com o Sistema Operacional Linux, através do cabo USB. Em seguida, carregamos o software Plot Spectrum desenvolvido, mostrado na Figura 3. Ao iniciar o software Plot Spectrum, automaticamente é configurada a frequência de $87,5 \mathrm{a} 108 \mathrm{MHz}$, logo, neste ponto, já temos todos os preparativos para a configuração do Analisador de Espectro e posteriormente obter dados para a comparação.

Figura 3 - Plot Spectrum após o carregamento das frequências.

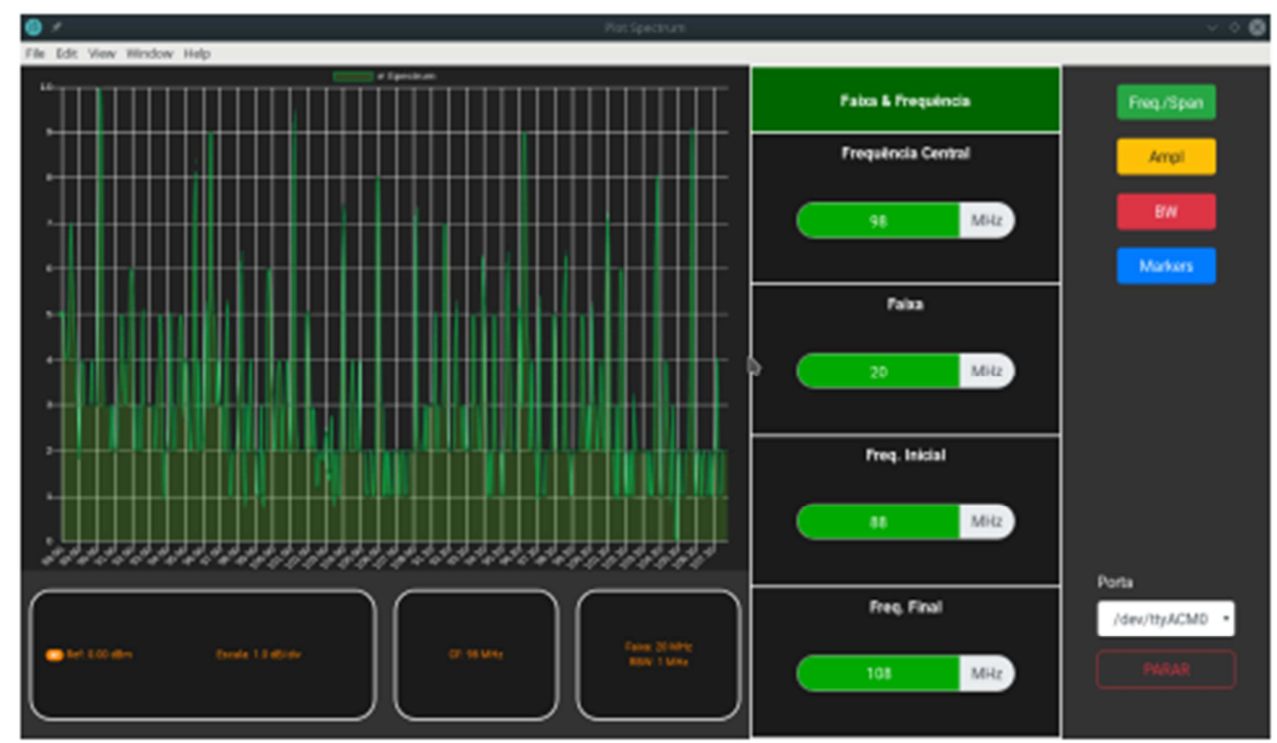

Fonte: Autores.

O Osciloscópio MDO3014 (mostrado na Figura 1) esteve com a função de Analisador de Espectro, onde configuramos a faixa de frequência de 87,5 a $108 \mathrm{MHz}$. A fim de obter estas frequências, utilizamos um gerador de sinais, o Tektronix AFG 3101 (Figura 4) e para receber os sinais, usamos uma antena de impedância de $50 \Omega$ (Figura 5). 


\section{COBENCE

Figura 4 - Gerador de Sinais Tektronix AFG 3101.

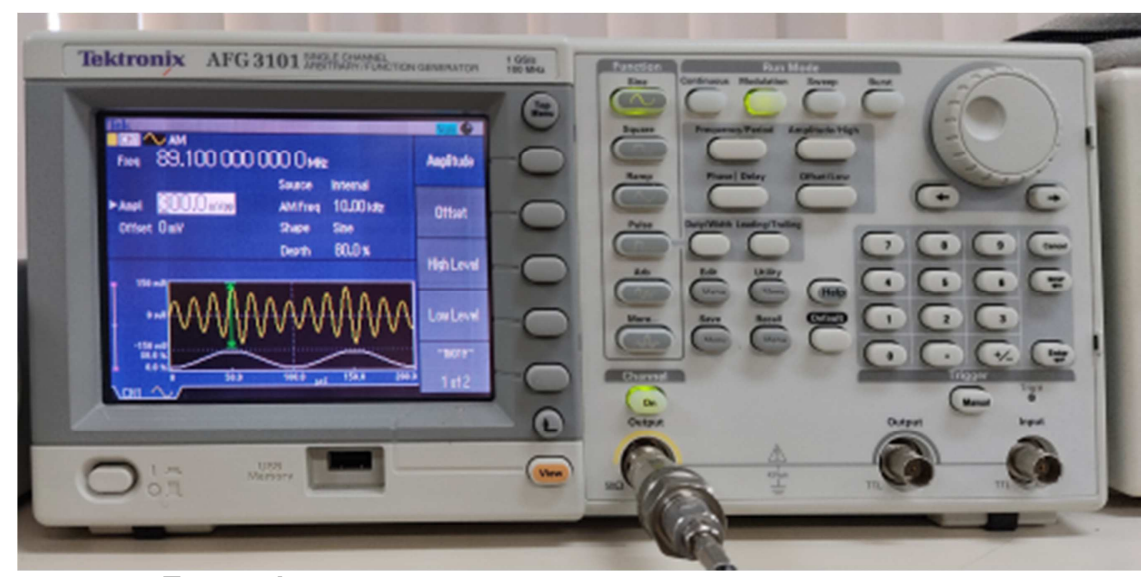

Fonte: Autores.

Figura 5 - Antena de 50 Ohms.

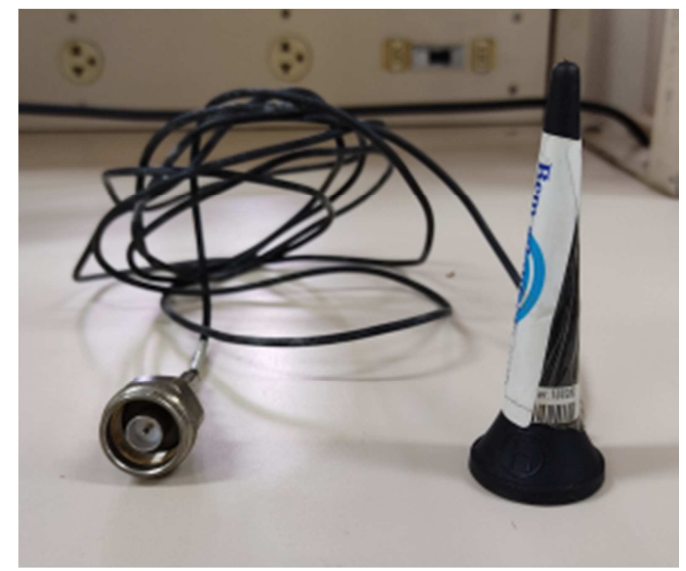

Fonte: Autores.

\section{Testes Realizados}

Realizamos 3 testes. Para o primeiro teste, na tela do AGF 3101, foi configurada a amplitude para o valor de $200 \mathrm{mVpp}$. Para o segundo e terceiro teste, repetimos os passos da configuração anterior, só alterando o valor para 300 e $500 \mathrm{mVpp}$, respectivamente. Todos os testes estão na mesma frequência, configuradas na tela inicial do gerador de sinais, em $89,1 \mathrm{MHz}$.

A primeira coleta dos dados consiste na captura de tela na faixa de 87,5 a $108 \mathrm{MHz}$ de ambos equipamentos analisados, do simulador e do analisador de espectro. Isso nos gera o resultado mostrado na Figura 6. Igualando-se os testes, devido à limitação do simulador, é configurada uma faixa de $1 \mathrm{MHz}$ em cima da frequência central $(89,1 \mathrm{MHz})$ do gerador de sinais, onde inicia em $88,6 \mathrm{MHz}$ e termina em $89,6 \mathrm{MHz}$. Com as três coletas registradas, sendo assim, para $200 \mathrm{mVpp}, 300 \mathrm{mVpp}$ e $500 \mathrm{mVpp}$, temos os resultados apresentados nas Figuras 7, 8 e 9. 


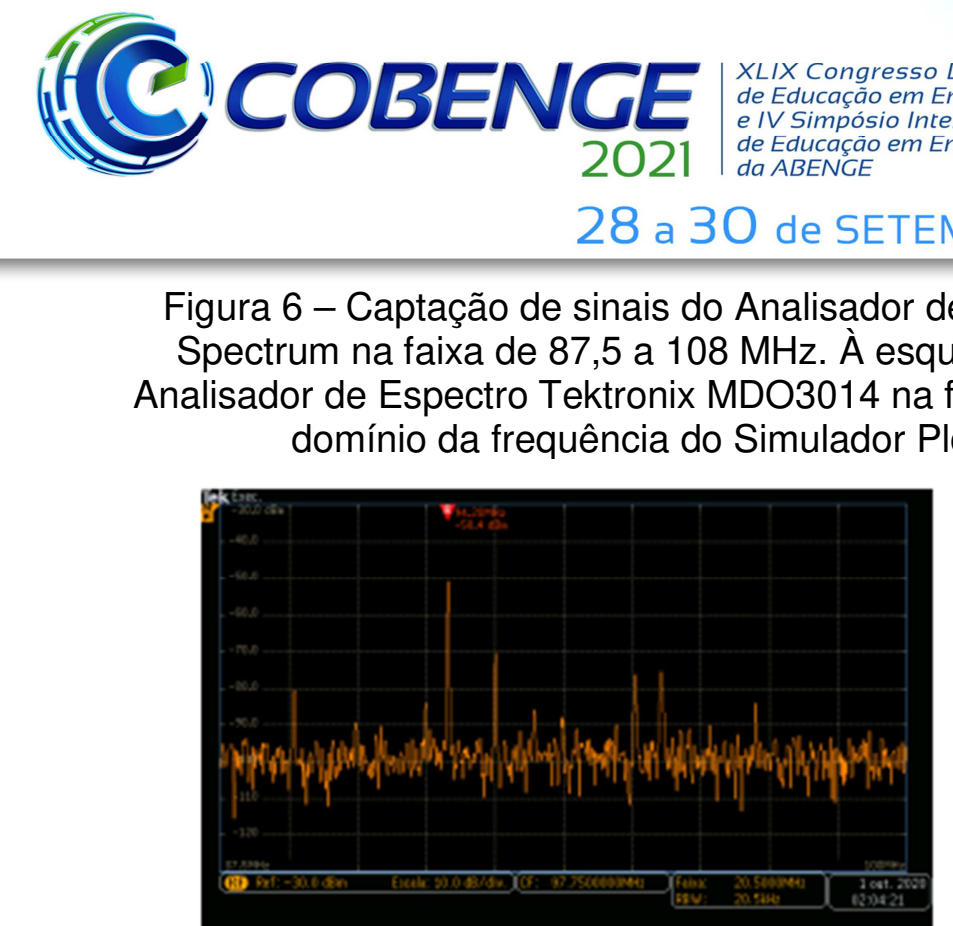

\section{Evento Online}

"Formação em Engenharia:

Tecnologia, Inovação e Sustentabilidade"

Figura 6 - Captação de sinais do Analisador de Espectro Tektronix MD03014 e Simulador Plot Spectrum na faixa de 87,5 a $108 \mathrm{MHz}$. À esquerda, visualização no domínio da frequência do Analisador de Espectro Tektronix MDO3014 na faixa de 87,5 a $108 \mathrm{MHz}$. À direita, visualização no domínio da frequência do Simulador Plot Spectrum na faixa de 87,5 a $108 \mathrm{MHz}$.

Fonte: Autores.

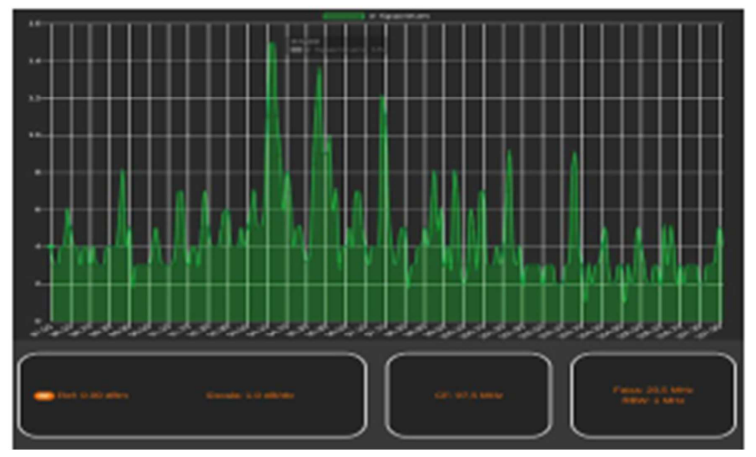

Figura 7 - Captação de sinais do Analisador de Espectro Tektronix MDO3014 e Simulador Plot Spectrum na faixa de 89,1 MHz em 200 mVpp do Gerador. À esquerda, visualização no domínio da frequência do Analisador de Espectro Tektronix MDO3014 na faixa de $89,1 \mathrm{MHz}$. À direita, visualização no domínio da frequência do Simulador Plot Spectrum na faixa de 89,1 MHz.
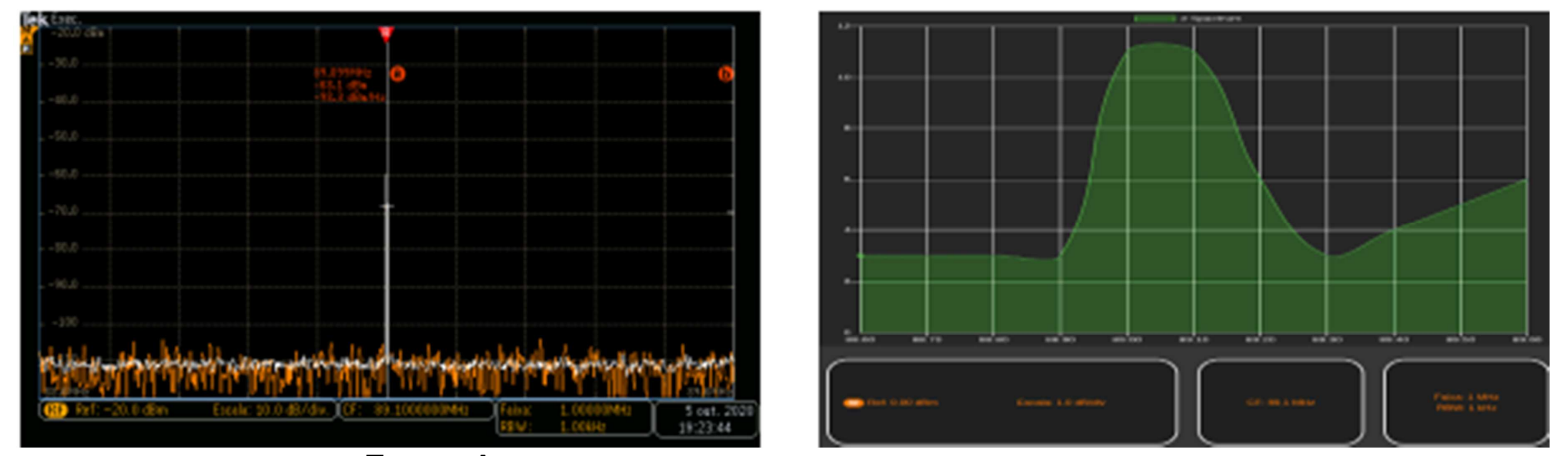

Fonte: Autores.

Figura 8 - Captação de sinais do Analisador de Espectro Tektronix MD03014 e Simulador Plot Spectrum na faixa de $89,1 \mathrm{MHz}$ em 300 mVpp do Gerador. À esquerda, visualização no domínio da frequência do Analisador de Espectro Tektronix MDO3014 na faixa de 89,1 MHz. À direita, visualização no domínio da frequência do Simulador Plot Spectrum na faixa de 89,1 MHz.
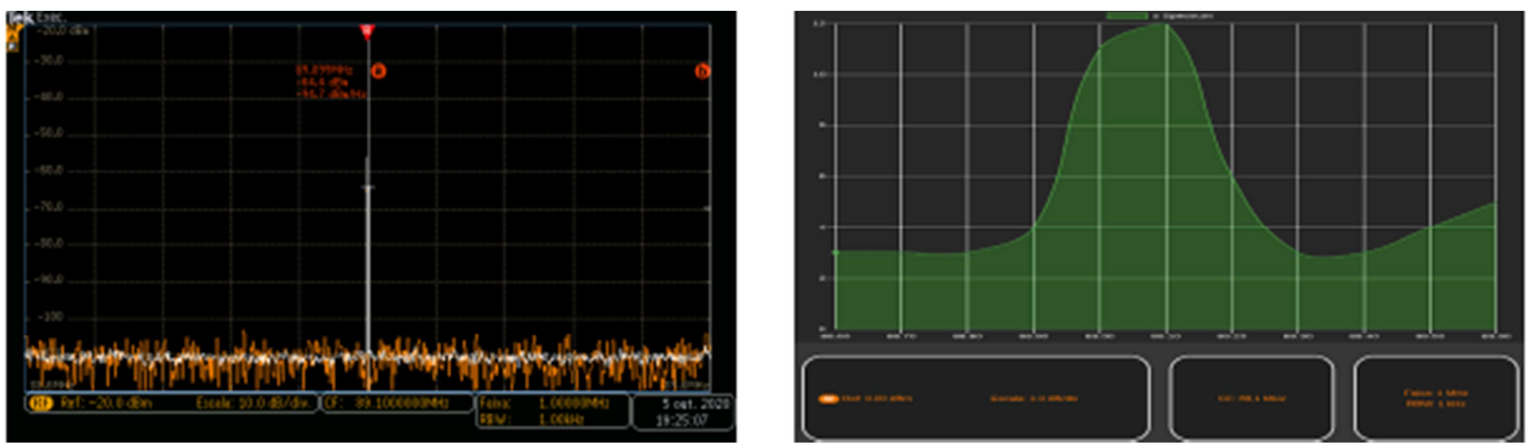

Fonte: Autores. 
Figura 9 - Captação de sinais do Analisador de Espectro Tektronix MDO3014 e Simulador Plot Spectrum na faixa de $89,1 \mathrm{MHz}$ em $500 \mathrm{mVpp}$ do Gerador. À esquerda, visualização no domínio da frequência do Analisador de Espectro Tektronix MDO3014 na faixa de $89,1 \mathrm{MHz}$. À direita, visualização no domínio da frequência do Simulador Plot Spectrum na faixa de $89,1 \mathrm{MHz}$.
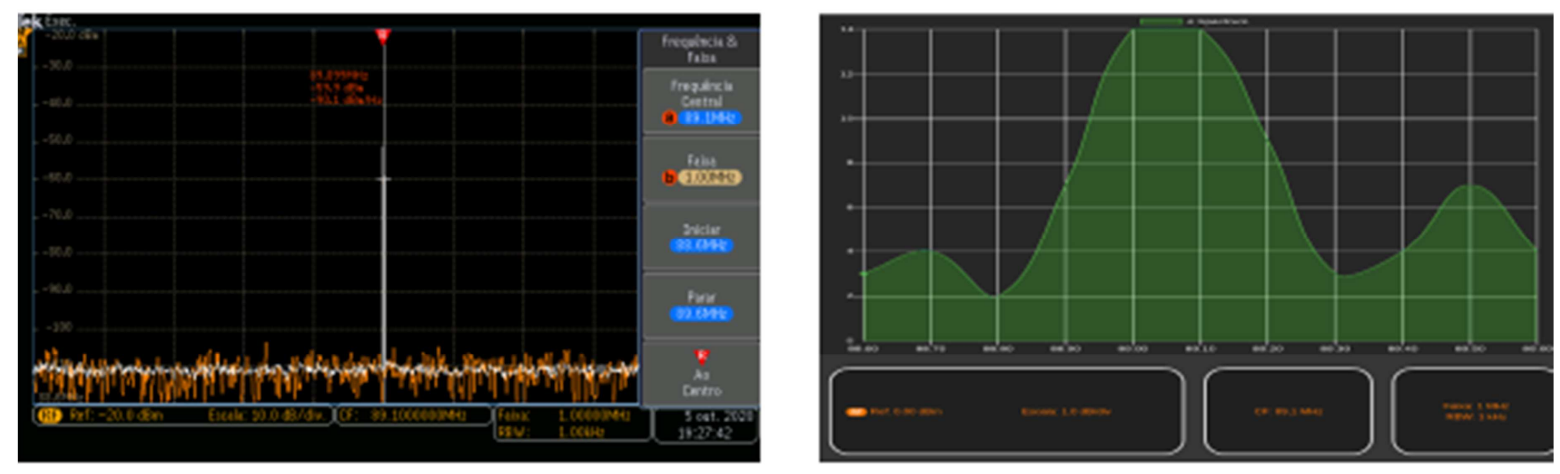

Fonte: Autores.

\subsection{Resultados}

Nesta seção, interpretamos os dados coletados das Figuras 6, 7, 8 e 9 para a análise dos resultados. Analisando a Figura 6, vemos muita semelhança nos gráficos, tanto no gráfico da esquerda quanto no da direita, onde há seis frequências em destaque em relação a potência das demais, que são frequências de estações de rádio da cidade Manaus, Amazonas: 89,7 MHz (Rádio Tiradentes FM), 94,3 MHz (FM do Povo), 95,1 MHz (Nativa FM), 97,5 MHz (Rádio Diário), 101,5 MHz (Rádio CBN Amazônia) e 103,5 MHz (Rádio Rio Mar), sendo a 94,3 MHz com maior potência com -50.4 dBm no Analisador de Espectro e $14 \mathrm{dBm}$ no Simulador. Interpretando os dados obtidos na frequência 89,1 $\mathrm{MHz}$, temos as Figuras 7, 8 e 9, mas para uma análise melhor dos valores em dBm, geramos a Tabela 1 a seguir.

Tabela 1 - Valores captados na frequência de $89,1 \mathrm{MHz}$ para diferentes tensões pico a pico captados via antena.

\begin{tabular}{c|c|c|c}
\hline $\mathrm{mVpp}$ (Volts) & Saída $(\mathrm{dBm})$ & Ent. Analisador $(\mathrm{dBm})$ & Ent. Simulador $(\mathrm{dBm})$ \\
\hline 200 & -10 & $-68,1$ & 11 \\
\hline 300 & $-6,48$ & $-64,4$ & 12 \\
\hline 500 & $-2,04$ & $-59,9$ & 14 \\
\hline
\end{tabular}

Fonte: Autores.

Com a Tabela 1, é interessante observar a diferença da potência de uma tensão pico a pico para outra em relação a saída no gerador, que é o que chamamos de potência relativa, ou seja, a diferença que ocorrer na saída do gerador deve ser a mesma diferença, ou bem próxima, captada pela antena do Analisador e do Simulador. Assim é elaborada uma nova tabela para melhor comparar tais potências, mostradas na Tabela 2.

Tabela 2 - Valores relativos de potência de uma tensão para

\begin{tabular}{c|c|c|c}
\hline \multicolumn{4}{c}{ outra. } \\
\hline mVpp (Volts) & Saída $(\mathrm{dBm})$ & Ent. Analisador (dBm) & Ent. Simulador (dBm) \\
\hline $200-300$ & $-3,52$ & $-3,7$ & -1 \\
\hline $300-500$ & $-4,44$ & $-4,5$ & -2 \\
\hline
\end{tabular}

Fonte: Autores.

\subsection{Comparação das Funcionalidades}

A comparação das funcionalidades (ditas anteriormente na Seção 2.1) é através das principais funções que engloba os conceitos de um analisador de espectro, que são: 
FFT: A validação do algoritmo FFT aplicado em ambos equipamentos analisados consiste na forma que o gráfico se dispõe em tela através da frequência e potência. Após toda a coleta da faixa de frequência de inicial a final na aplicação, o Plot Spectrum "desenha" todas as informações em forma de gráficos, chegando ao resultado final apresentado anteriormente na Figura 3. Já o Analisador de Espectro tem um comportamento diferente na varredura. Ele capta todos os dados de uma vez e "desenha" na tela dependendo da resolução adotada, onde no teste foi configurada uma resolução automática.

SPAN: O SPAN faz a amostra visual de uma largura de banda já coletada, define o que será mostrado nas frequências de inicio e fim sem depender diretamente dos dados coletados na largura de banda. Em outras palavras, compreende toda a área do gráfico tanto no Analisador de Espectro quanto no Simulador. A maior diferença entre os dois é que no simulador é feito de forma automática, podendo ser modificado somente via código até a implementação dessa funcionalidade, $\mathrm{e}$ no Analisador pode ser configurada manualmente e de forma automática. A forma automática dos dois equipamentos dependem diretamente da frequência central e da largura de banda, sendo assim, não interfere na forma de visualizar dos dados na tela.

Frequência central: A frequência central é exatamente o meio da faixa em que está sendo coletado os dados. A Figura 3 mostra todos os pontos que esta frequência pode ser identificada no Plot Spectrum. No Analisador de Espectro também temos esses indicadores em tela como mostram as Figuras 6, 7, 8 e 9.

Nível de referência: $O$ nível de referência é $0 \mathrm{dBm}$ para o simulador Plot Spectrum devido a limitação da placa TEA5767 e a forma que o driver foi escrito. O limite do analisador do espectro depende muito da antena utilizada e potência transmitida, mas como da saída do gerador até a captura do sinal pela antena sempre tem perda, é normal trabalhar com valores negativos, sendo valores legíveis de $5 \mathrm{MHz}$ a 2 $\mathrm{GHz}$ de até $-138 \mathrm{dBm} / \mathrm{Hz}$, segundo o manual do fabricante.

Escala: A escala dos gráficos do simulador é dinâmica, mostra de acordo com a configuração do SPAN, se adaptando a tela. Em contrapartida, a escala do analisador de espectro é estática e pode ser configurada de acordo com o que o usuário definir.

RBW: O RBW utilizado pelo simulador é de $15 \mathrm{KHz}$, limitação da placa TEA5767, mas para a varredura não demorar tanto é colocado $100 \mathrm{KHz}$ por padrão, podendo ser alterado via código. No analisador de espectro pode ser configurado até $1 \mathrm{KHz}$ de acordo com o manual. Nesta análise, o analisador de espectro recebeu uma resolução automática, pois resoluções manuais afetam seu desempenho.

Marcadores: A funcionalidade dos marcadores no simulador é através do mouse, onde basta passá-lo por cima do ponto que deseja ser analisado. No analisador de espectro é feito de maneira diferente, onde pode-se marcar o ponto "a" e "b" para visualizar melhor a frequência e a potência em $\mathrm{dBm}$.

\subsection{Comparação com os Trabalhos Relacionados}

Em comparação a primeira fonte citada, este trabalho acadêmico tem intuito de apresentar um simulador de um analisador de espectro como alternativa viável para aprendizagem e não tem como objetivo usar uma ferramenta já existente para o desenvolvimento de interfaces, pelo contrário, é desenvolvimento com linguagem WEB, teoricamente fácil de trabalhar, onde o código fonte é aberto, ou seja, qualquer um pode ajudar a distribuir, copiar e modificar. Outro ponto comparativo é que apesar de trabalhar de forma indireta com a Transformada de Fourier, este trabalho não teve como foco a precisão dos dados comparados, mas sim a facilitação do manuseio de um equipamento de difícil acesso. Comparando a segunda fonte, este trabalho tem frequências específicas 
de rádio $\mathrm{FM}$, onde não inclina para modulações de sinais. Frequências estas escolhidas pela sua grande difusão aérea, a qual pode ser capturada em qualquer momento, permitindo trabalhar com dados reais com facilidade. Além disso, não direciona apenas em modulação, sinal modulante e portadora, mas em faixas de frequências capturadas por um módulo Arduino. A diferença deste trabalho para a terceira fonte é que apesar de utilizarem o MATLAB, o trabalho de Melo et al. (2019) não tem interface interativa e não é código aberto. No nosso trabalho não é utilizado conversores $A / D$ de forma direta como no terceiro trabalho, pois a ideia foi facilitar o funcionamento do equipamento e não entrar em detalhes de como construí-lo, mas como proposta traz a conversão já adaptada por meio do Arduino e a placa de rádio FM (shield).

\section{Conclusão}

Em resumo, apesar todos os Trabalhos Relacionados trabalharem com frequências, possuem propostas semelhantes para facilitar disciplinas difíceis, mas como principal diferencial é que o nosso trabalho elaborou uma ferramenta desde a sua concepção inicial que está disponível em uma plataforma de versionamento online, onde qualquer pessoa com acesso à Internet pode ter acesso ao código fonte. A vantagem de ter acesso ao código fonte é que este pode ser estudado, modificado ou distribuído como convier, respeitando a licença de código aberto utilizada. Outro diferencial no nosso trabalho foi também de utilizar hardwares prontos e de fácil acesso e baixo custo (Arduino e shield TEA5767). Além disso, o Plot Spectrum simula um analisador de espectro com dados reais, onde podem ser explorados todos os conceitos das disciplinas que utilizam este equipamento, em especial a disciplina de Micro-ondas do curso de Telecomunicações.

Com base nos experimentos realizados, pode-se concluir que, em relação a faixa total de 87,5 a $108 \mathrm{MHz}$, os dois equipamentos apresentam gráficos bastante semelhantes e na frequência correta. Ainda na faixa total, a potência tem diferenças onde 0 analisador trabalha em potência de 0 a $-138 \mathrm{dBm}$ e o simulador trabalha de $0 \mathrm{a}+15$ $\mathrm{dBm}$ (potência invertida), dificultando a comparação entre os dois. A visualização das frequências em faixas pequena acabam afetam a plotagem do gráfico tornando-a mais difícil para comparação com o analisador de espectro, porém estes detalhes podem ser ajustado via programação. Em relação a abstração das potências relativas apresentadas no Tabela 2, podemos concluir que o analisador alcança a valores próximos em relação ao de saída do gerador de sinais, enquanto o simulador fica com valores distantes, não permitindo compará-los com o analisador.

Com todos esses pontos abordados, podemos concluir que Simulador Plot Spectrum, embora tenha muitas limitações, é focado principalmente nas funcionalidades básicas de um analisador de espectro real, sendo uma alternativa viável para o que se propõe, contendo um potencial para o incentivo do desenvolvimento de software e melhorias futuras para esta aplicação, e que pode ser usado para fins didáticos.

\section{REFERÊNCIAS}

FERRER, V. Analizador de espectro. 2019. Disponível em: https://vicentferrer.com/analizador-de-espectro. Acesso em: 21 de out de 2019.

FRANCHI, T. P. et al. Desenvolvimento de uma ferramenta computacional acadêmica para estudo da modulação am-dsb/sc. Anais do COBENGE 2018. Disponível em: http://www.abenge.org.br/cobenge/. Acesso em: 6 de set de 2020. 
GUEDES, D. L.; GÓES, D. B. R.; SILVA, O. F. Desenvolvimento de interface gráfica em matlab para aprimorar o ensino na disciplina de processamento digital de sinais. Anais do COBENGE 2016. Disponível em: http://www.abenge.org.br/cobenge/. Acesso em: 30 de set de 2020 .

MELO, P. H. B. et al. Projeto multidisciplinar de um analisador de espectro com bancos de filtros e subamostragem. Anais do COBENGE 2019. Disponível em: http://www.abenge.org.br/cobenge/. Acesso em: 17 de set de 2020.

TEKTRONIX. MDO4000C Mixed Domain Oscilloscope. Disponível em: https://www.tek.com/oscilloscope/mdo4000c-mixed-domain-oscilloscope. Acesso em: 04 mai. 2021.

\title{
A SPECTRUM ANALYZER WITH ARDUINO ON THE RADIO BAND FM FOR TEACHING PURPOSES
}

\begin{abstract}
This article presents the development of software, called Plot Spectrum, which simulates a Spectrum Analyzer for didactic purposes in Telecommunications course subjects made with Arduino and a TEA5767 shield of FM radio in the frequency of 87.5 to $108 \mathrm{MHz}$ interacting with any personal computer. In addition, the project features are presented comparing with a real Spectrum Analyzer, or an Oscilloscope with compatible function, and also comparing with similar projects already published.
\end{abstract}

Keywords: Spectrum Analyzer. Arduino. Simulator. 